\title{
TPR-TNR plot for confusion matrix
}

\author{
Chong Sun Hong ${ }^{1, a}$, Tae Gyu Oh ${ }^{a}$ \\ ${ }^{a}$ Department of Statistics, Sungkyunkwan University, Korea
}

\begin{abstract}
The two-dimensional confusion matrix used in credit assessment, biostatistics, and many other fields consists of true positive, true negative, false positive, and false negative. Their rates, such as the true positive rate (TPR), true negative rate (TNR), false positive rate, and false negative rate, can be applied to measure its accuracy. In this study, we propose the TPR-TNR plot, a graphical method that can geometrically describe and explain these rates based on the confusion matrix. The proposed TPR-TNR plot consists of two right-angled triangles. We obtain that the TPR and TNR describe the acute angles of right-angled triangles in the plot. These acute angles can be used to determine optimal thresholds corresponding to lots of accuracy measures.
\end{abstract}

Keywords: accuracy, confusion, triangle, threshold.

\section{Introduction}

Statistical classification results can be expressed by the $2 \times 2$ confusion matrix shown in Table 1 for various areas such as biostatistics and credit evaluation. The true positive (TP) and true negative (TN) in Table 1 represent the number of correctly classified disease and normal populations, respectively. In other words, the TP is the number of patient with an actual disease will be positive, and the TN is the number of normal people to be negative. However, the false positive (FP) and false negative (FN) are numbers of groups that predicted disease as normal and predicted normal as disease, respectively (Metz and Kronman, 1980; Swets, 1988; Hsieh and Turnbull, 1996; Provost and Fawcett, 1997, 2001; Engelmann et al., 2003; Pepe, 2003; Fawcett, 2004, 2006; Stein, 2005; Sonego et al., 2008; Hong et al., 2010; Pontius and Si, 2014; Cho and Hong, 2015; Hong and Lee, 2018; Oehr and Ecke, 2020).

The following probability equations express the true positive rate (TPR) and true negative rate (TNR). It is worth knowing that the TPR and TNR are also known as sensitivity and specificity, respectively (Altman and Bland, 1994).

$$
\begin{aligned}
& \mathrm{TPR}=\text { sensitivity }=\frac{\mathrm{TP}}{\mathrm{TP}+\mathrm{FN}}, \\
& \mathrm{TNR}=\text { specificity }=\frac{\mathrm{TN}}{\mathrm{TN}+\mathrm{FP}} .
\end{aligned}
$$

Let the parameter space $\theta$ represent the patient states and be assumed to consist of two elements, $\left\{\theta_{d}, \theta_{n}\right\}$, where $\theta_{d}$ and $\theta_{n}$ mean disease/default/positive and non-disease/non-default/negative states, respectively. For a random variable $X$, the conditional cumulative distribution functions of $X$ given disease and non-disease states are denoted by $F_{d}(x)=P\left(X \leq x \mid \theta_{d}\right)$ and $F_{n}(x)=P\left(X \leq x \mid \theta_{n}\right)$,

\footnotetext{
${ }^{1}$ Corresponding author: Department of Statistics, Sungkyunkwan University, 25-2, Sungkyunkwan-ro, Jongno-gu, Seoul 03063, Korea. E-mail: cshong@skku.edu
}

Published 31 March 2021 / journal homepage: http://csam.or.kr

(c) 2021 The Korean Statistical Society, and Korean International Statistical Society. All rights reserved. 
Table 1: Confusion matrix

\begin{tabular}{cccc}
\hline \hline & & \multicolumn{2}{c}{ Predicted } \\
\cline { 3 - 4 } & & Disease & \multicolumn{2}{c}{ Non-Disease } \\
\hline \multirow{2}{*}{ Actual } & Disease & TP & FN \\
\cline { 2 - 4 } & Non-Disease & FP & TN \\
\hline \hline
\end{tabular}

respectively. And generally suppose that $F_{d}(x) \geq F_{n}(x)$ for all $x$ (Mets and Kronman, 1980; Hsieh and Turnbull, 1996; Provost and Fawcett, 2001; Engelmann et al., 2003; Pepe, 2003; Fawcett, 2006). Then the TPR and TNR are defined by TPR $=F_{d}(x)$ and TNR $=1-F_{n}(x)$, respectively. Similarly, the false positive rate (FPR) and false negative rate (FNR) are defined as FPR $=F_{n}(x)$ and FNR $=1-F_{d}(x)$, respectively.

Based on statistical decision theory, the receiver operating characteristic (ROC) curve is a visual tool that can easily identify the classifier's performance in binary classification (Green and Swets, 1966; Bamber, 1975; Egan and Egan, 1975; Hanley and McNeil, 1982; Swets, 1988; Centor, 1991; Zweig and Campbell, 1993; Fawcett, 2004; Vuk and Curk, 2006; Tasche, 2008). Since the ROC curve is implemented with ( $1-\mathrm{TNR}$, TPR) in a unit length square, the TPR and TNR can be represented on the ROC curve.

In this study, we propose a graphical method to describe the TPR and TNR geometrically. The geometric representation consists of two right-angled triangles based on two sum equations of the confusion matrix: TPR + FNR $=1$, FPR + TNR $=1$. This graphical method is called the TPR-TNR plot, representing the TPR and TNR expressed geometrically on an upper half-circle. The TPR-TNR plot is explored in terms of many accuracy measures for finding optimal thresholds (cutoff points). Some characteristics of the TPR-TNR plot are derived with several distribution functions.

The remainder of the paper is organized as follows. Section 2 introduces the TPR-TNR plot, describing the geometric representation of the TPR, TNR, FPR, and FNR. Particularly, the TPR and TNR are definded as functions of acute angles of right-angled triangles. The shapes, locations, and distance of two right-angled triangles on an upper half-circle in the TPR-TNR plot describe the discriminant power of two data sets. In Section 3, we explain three strategies for finding optimal thresholds using the functions of two acute angles of the right-angled triangles. The first strategy is to maximize the summation of the two acute angles. The second strategy is to maximize the product of two acute angles, and the last strategy is to minimize the absolute value of the difference between the two acute angles. We obtain that these methods have some relationship with many accuracy measures. In Section 4 , we present various normal distribution functions to derive the TPR-TNR plot's characteristics so that two right-angled triangles in the TPR-TNR plot could geometrically explain a confusion matrix. In Section 5, we illustrate the empirical data and describe the TPR-TNR plot based on the confusion matrix. Section 6 presents the conclusions.

\section{TPR-TNR plot}

With an equation $A+B=1$, one can draw a right-angled triangle with two short line segments of lengths $\sqrt{A}$ and $\sqrt{B}$, and one longest line segment of length 1. From the confusion matrix in Table 1, the following three equations are obtained

$$
\mathrm{TPR}+\mathrm{FNR}=1, \quad \mathrm{FPR}+\mathrm{TNR}=1 .
$$

Two right-angled triangles in Figure 1 can be implemented from two equations in (2.1). This plot is based on the confusion matrix in Table 2 . 
Table 2: An example of confusion matrix

\begin{tabular}{cccc}
\hline \hline & & \multicolumn{2}{c}{ Predicted } \\
\cline { 3 - 4 } & & Disease & Non-Disease \\
\hline \multirow{2}{*}{ Actual } & Disease & 80 & 20 \\
\cline { 2 - 4 } & Non-Disease & 24 & 101 \\
\hline \hline
\end{tabular}

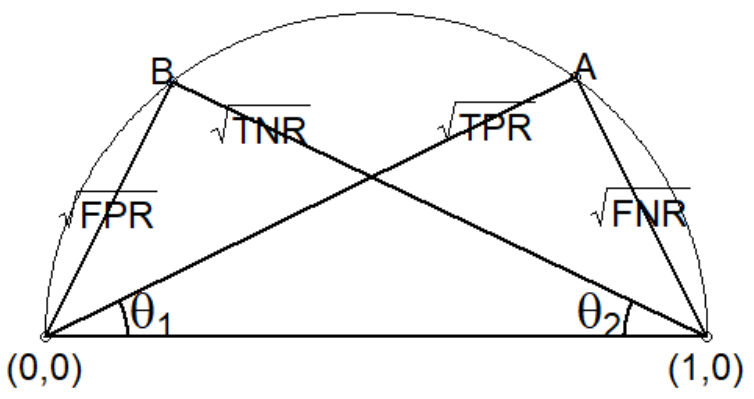

Figure 1: TPR-TNR plot.

From Figure 1, the length of the horizontal line is 1 . The rightmost and leftmost segments are represented by $\sqrt{\mathrm{FNR}}$ and $\sqrt{\mathrm{FPR}}$, respectively. Therefore, $\theta_{1}$ and $\theta_{2}$ correspond to the right and left right-angled triangles, respectively.

Remark 1. The two acute angles $\theta_{1}$ and $\theta_{2}$ in Figure 1 are defined by the following equations:

$$
\cos ^{2} \theta_{1}=\mathrm{TPR}, \quad \cos ^{2} \theta_{2}=\mathrm{TNR} .
$$

Also FNR $=\cos ^{2}\left(\pi / 2-\theta_{1}\right)$ and FPR $=\cos ^{2}\left(\pi / 2-\theta_{2}\right)$. Hence, $\theta_{1}=\cos ^{-1} \sqrt{\mathrm{TPR}}$ and $\theta_{2}=$ $\cos ^{-1} \sqrt{\mathrm{TNR}}$. Moreover, $\theta_{1}=\sin ^{-1} \sqrt{\mathrm{FNR}}, \theta_{2}=\sin ^{-1} \sqrt{\mathrm{FPR}}$. Therefore, the TPR and TNR are described by two angles $\theta_{1}$ and $\theta_{2}$, so do the FPR and FNR in Figure 1.

Let $A$ be the point where the sides corresponding to the TPR and FNR meet in the right triangle, and let $B$ be the point where the sides corresponding to the FPR and TNR meet in the left triangle. We obtain that both points $A$ and $B$ are on the upper half-circle, and point $A$ precedes point $B$.

Remark 2. The coordinates of point $A$ and $B$ are obtained, such as:

$$
\begin{aligned}
& A=(\mathrm{TPR}, \sqrt{\mathrm{TPR} \mathrm{FNR}})=\left(F_{d}(x), \sqrt{F_{d}(x)\left(1-F_{d}(x)\right)}\right), \\
& B=(\mathrm{FPR}, \sqrt{\mathrm{FPR} \mathrm{TNR}})=\left(F_{n}(x), \sqrt{F_{n}(x)\left(1-F_{n}(x)\right)}\right) .
\end{aligned}
$$

If both points $A$ and $B$ locate very closely, then the discriminant power is very poor. On the other hand, in the case that the point $A$ locates close to a coordinate $(1,0)$ and the point $B$ locates close to the origin $(0,0)$, then two different data sets are classified almost perfectly. Therefore, it might be said that the farther point $A$ is away from point $B$, the easier it is to classify the two data sets. Hence, the distance between $A$ and $B$ points plays an important role to classify.

As both points $A$ and $B$ move from the origin to a coordinate $(1,0)$, the angle $\theta_{1}$ corresponding to point $A$ decreases from $\pi / 2$ to 0 , while the angle $\theta_{2}$ corresponding to the point $B$ increases from 
0 to $\pi / 2$. Also, it is found that as the random variable $X$ increases from $-\infty$ to $\infty, \cos ^{2} \theta_{1}$ increases while $\cos ^{2} \theta_{2}$ decreases. From Table 2 and Figure 1 , we obtain that TPR $=0.8000$, TNR $=0.8080$, $\theta_{1}=26.5651^{\circ}$ and $\theta_{2}=25.9877^{\circ}$. Since the TPR and TNR have large values, two angles of $\theta_{1}$ and $\theta_{2}$ are similar and have small values.

\section{Optimal thresholds on TPR-TNR plot}

To determine optimal thresholds, many accuracy measures used the TPR and TNR, which are defined as $\cos ^{2} \theta_{1}$ and $\cos ^{2} \theta_{2}$ on Remark 1. Some of them are the Youden index (J: Youden, 1950; Fluss et al., 2005; Perkins and Schisterman, 2005), the maximum vertical distance (MVD: Krzanowski and Hand, 2009), the sum of sensitivity and specificity (SSS: Connell and Koepsell, 1985), the true rate which is an average of the TPR and TNR (TR: Hong et al., 2010), the amended closet-to-(0,1) (AC: Pepe, 2003; Perkins and Schisterman, 2006), the maximizing the accuracy area or the product of sensitivity and specificity (AA: Brasil, 2010; Liu, 2012), and the symmetric point (SP: Moses et al., 1993; Pepe, 2003). Therefore, two acute angles $\cos ^{2} \theta_{1}$ and $\cos ^{2} \theta_{2}$ on the TPR-TNR plot could be applied to determine optimal thresholds using the following three remarks.

Remark 3. An optimal threshold can be obtained by satisfying the following:

$$
\max \left\{\cos ^{2} \theta_{1}+\cos ^{2} \theta_{2}\right\} \text {. }
$$

Since the summation of $\cos ^{2} \theta_{1}$ and $\cos ^{2} \theta_{2}$ means the sum of the true positive and true negative ratios, it is found that the maximization of this summation is equivalent to $\max \left\{F_{d}(x)-F_{n}(x)\right\}$. Therefore, the maximization of the summation of $\cos ^{2} \theta_{1}$ and $\cos ^{2} \theta_{2}$ plays an important role to evaluate the discriminant power and determine optimal thresholds based on many accuracy measures such as the J, MVD, SSS, TR, AC accuracy measures. These measures are linear functions of the well-known Kolmogorov-Smirnov statistic (Hong and Yoo, 2011). Among the five measures, the $\mathrm{J}$ is used as a representative measure for Remark 3.

Remark 4. An optimal threshold can also be determined by satisfying the following:

$$
\max \left\{\cos ^{2} \theta_{1} \times \cos ^{2} \theta_{2}\right\} .
$$

Since the product of $\cos ^{2} \theta_{1}$ and $\cos ^{2} \theta_{2}$ is equivalent to the product of the true positive and true negative ratios: $\max \left\{F_{d}(x) \times\left(1-F_{n}(x)\right)\right\}$, the maximization this product is used to find an optimal threshold based on the accuracy area (AA) accuracy measure.

Remark 5. Another optimal threshold could be found by satisfying the following:

$$
\min \left|\cos ^{2} \theta_{1}-\cos ^{2} \theta_{2}\right| \text {. }
$$

When the absolute value of the difference between $\cos ^{2} \theta_{1}$ and $\cos ^{2} \theta_{2}$ is zero on the TPR-TNR plot, it can be said that $\theta_{1}=\theta_{2}$, so that the TPR is supposed to be equal to the TNR: $F_{d}(x)=1-F_{n}(x)$. Hence, the minimization of this absolute value is used to find an optimal threshold based on the symmetric point (SP) accuracy measure.

Note that when the heights of two points $A$ and $B$ on the TPR-TNR plot are the same, i.e., $F_{d}(x)(1-$ $\left.F_{d}(x)\right)=F_{n}(x)\left(1-F_{n}(x)\right)$, then this condition could also be equivalent to the SP, which also satisfies 


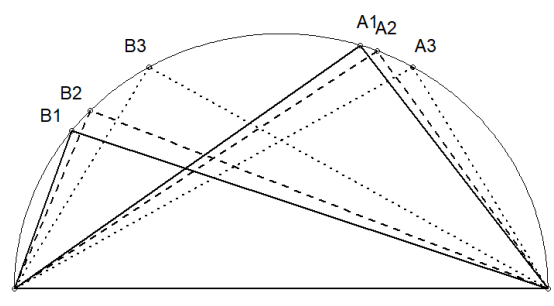

(a) $\mu_{n}=1, \sigma_{n}=0.5$

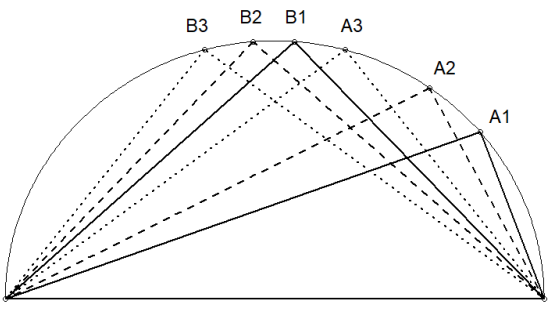

(b) $\mu_{n}=1, \sigma_{n}=2$

Figure 2: Optimal thresholds on TPR-TNR plots.

Remark 5. Therefore, we conclude that various optimal thresholds could be determined using $\theta_{1}$ and $\theta_{2}$ on the TPR-TNR plot.

Let points $A 1$ and $B 1$ correspond to the $\mathrm{J}$, points $A 2$ and $B 2$ correspond to the $\mathrm{AA}$, and points $A 3$ and $B 3$ correspond to the SP on the TPR-TNR plot. The distribution function of a disease state is assumed as the standard normal distribution $N(0,1)$, and two kinds of distributions of a non-disease state are considered: one is $\mu_{n}=1, \sigma_{n}=0.5$ and the other is $\mu_{n}=1, \sigma_{n}=2$. For the first case, it is obtained that optimal thresholds corresponding to the Youden, AA and SP are $x_{\mathrm{J}}=0.38, x_{\mathrm{AA}}=0.46$, $x_{\mathrm{SP}}=0.66$, respectively $\left(x_{\mathrm{J}}<x_{\mathrm{AA}}<x_{\mathrm{SP}}\right)$. Then from Figure 2(a), the horizontal coordinates of points $A$ and $B$ are satisfying that $B 1<B 2<B 3$ and $A 1<A 2<A 3$, since $\theta_{1}$ and the TNR decrease whereas $\theta_{2}$ and the TPR increase. For the second case in Figure 2(b), it is obtained that optimal thresholds corresponding to the Youden, AA and SP are $x_{\mathrm{J}}=1.18, x_{\mathrm{AA}}=0.79, x_{\mathrm{SP}}=0.33$, respectively, $\left(x_{\mathrm{J}}>x_{\mathrm{AA}}>x_{\mathrm{SP}}\right)$. Then, the horizontal coordinates of points $A$ and $B$ on Figure 2(b) are satisfying that $B 3<B 2<B 1$ and $A 3<A 2<A 1$, since $\theta_{1}$ and the TNR increase whereas $\theta_{2}$ and the TPR decrease. Moreover, the TPRs for the first case are smaller than those of the second case but the TNRs for the first case are larger than those of the second case based on three kinds of accuracy measures. It if found that the points $A$ for the second case in Figure 2(b) locate nearer to $(1,0)$ than the first case in Figure 2(a), and the points $B$ for the first case in Figure 2(a) locate closer to $(0,0)$ than the first case in Figure 2(b).

\section{Properties of TPR-TNR plot}

In order to examine the properties of the TPR-TNR plot, we consider the normal distribution functions with various means and variances to understand the TPR-TNR plot's characteristics. For each distribution function, the confusion matrix is obtained for the optimal threshold based on the $\mathrm{J}$ among the accuracy measures discussed in Section 3. The TPR-TNR plots corresponding these confusion matrices are explored.

We obtain that the conditional cumulative distribution functions given a disease state $F_{d}(x)$ follows a standard normal distribution $N\left(0,1^{2}\right)$ and the conditional cumulative distribution functions given a non-disease state $F_{n}(x)$ follows normal distributions $N\left(\mu_{n}, \sigma^{2}\right)$ with $\mu_{n}=1,2$ and $\sigma_{n}=0.5,1,1.5$. For the four types of $F_{n}(x)$ in Table 3, the optimal threshold and $\theta_{1}, \theta_{2}$ are calculated based on the $\mathbf{J}$. Figure 3 shows the four TPR-TNR plots with these values.

Using Figure 3, we obtain that when $\mu_{n}$ is greater than $\mu_{d}$ with fixed $\sigma_{d}=\sigma_{n}$, the two angles $\theta_{1}$ and $\theta_{2}$ decrease, leading to an increase in the TPR and TNR. Therefore, the two points $A$ and $B$ spread farther apart on half upper-circle when $\mu_{n}$ is greater than $\mu_{d}$ (see Figure 3(a) and (b)). Moreover, when $\sigma_{n}=1.5$ is greater than $\sigma_{d}$ with $\mu_{d}=\mu_{n}, \theta_{1}$ decreases and $\theta_{2}$ increases, leading to an increase and decrease in the TPR and TNR, respectively. Therefore, the two points $A$ and $B$ are located on the right 
Table 3: TPR and TNR for various distributions

\begin{tabular}{lccccc}
\hline \hline & Optimal threshold & TPR & TNR & $\theta_{1}$ & $\theta_{2}$ \\
\hline (a) $\mu_{n}=1, \sigma_{n}=1$ & 0.5000 & 0.6915 & 0.6915 & $33.7425^{\circ}$ & $33.7425^{\circ}$ \\
(b) $\mu_{n}=2, \sigma_{n}=1$ & 1.0000 & 0.8413 & 0.8413 & $23.4729^{\circ}$ & $23.4729^{\circ}$ \\
(c) $\mu_{n}=1, \sigma_{n}=1.5$ & 0.9028 & 0.8167 & 0.5258 & $25.3496^{\circ}$ & $43.5199^{\circ}$ \\
(d) $\mu_{n}=1, \sigma_{n}=0.5$ & 0.3812 & 0.6485 & 0.8921 & $36.3629^{\circ}$ & $19.1795^{\circ}$ \\
\hline \hline
\end{tabular}

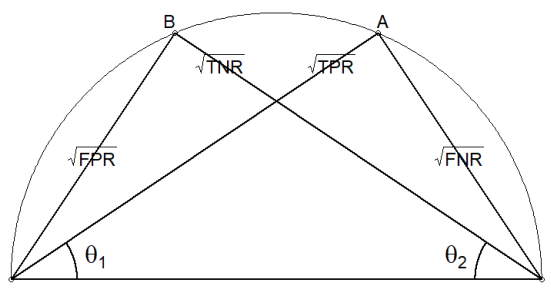

(a) $\mu_{n}=1, \sigma_{n}=1$

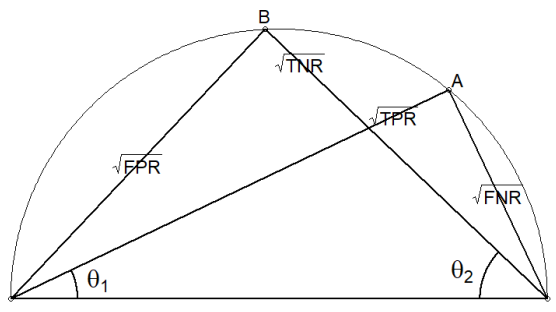

(c) $\mu_{n}=1, \sigma_{n}=1.5$

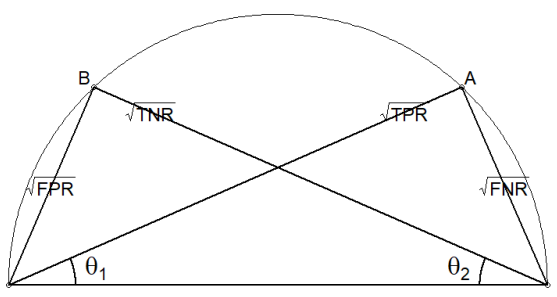

(b) $\mu_{n}=2, \sigma_{n}=1$

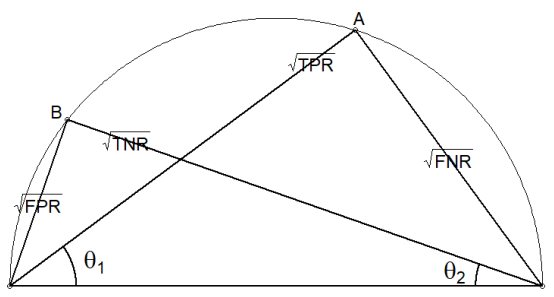

(d) $\mu_{n}=1, \sigma_{n}=0.5$

Figure 3: TPR-TNR plots.

of the half upper-circle when $\sigma_{n}$ is greater than $\sigma_{d}$ (see Figure 3(a) and (c)). From Figure 3(d), we have that when $\sigma_{n}=0.5$ is less than $\sigma_{d}$ with $\mu_{d}=\mu_{n}$, values of the $\theta_{1}$ and $\theta_{2}$ are in contrast to the case of Figure 3(c) leading to a decrease and increase in the TPR and TNR, respectively. Therefore, the two points $A$ and $B$ are located on the left of the half upper-circle (see Figure 3(c) and (d)).

\section{Real data example}

A credit evaluation data was collected from a Korean domestic K bank in June 2018. This sample of size 65,455 contains two random variables: one means the risk score rank, which is a score variable with 20 grades, and the other variable divides each sample into three stages. Hong and Choi (2020) used the second and third stages of size $q=6,951(C=1)$ and $q=1,191(C=0)$, respectively. These two stages are classified into non-default and default groups. Hong and Choi (2020) obtained the same $2 \times 2$ confusion matrix in Table 4 based on an optimal threshold.

From Table 4, we obtain that TPR $=$ sensitivity $=0.9501$ and TNR $=$ specificity $=0.8371$. These values are almost close to 1.0, which means that the accuracy and performance of the diagnosis are very superior. Figure 4 shows the TPR-TNR plot for Table 4 . From Figure 4 , we obtain that $\theta_{1}=12.9106^{\circ}$ is smaller than $\theta_{2}=23.8031^{\circ}$ since the TPR has larger value than the TNR. 
Table 4: Confusion matrix for an empirical data

\begin{tabular}{cccc}
\hline \hline & & \multicolumn{2}{c}{ Predicted } \\
\cline { 3 - 4 } & & Disease & Non-Disease \\
\hline \multirow{2}{*}{ Actual } & Disease & 6604 & 347 \\
\cline { 2 - 4 } & Non-Disease & 194 & 997 \\
\hline \hline
\end{tabular}

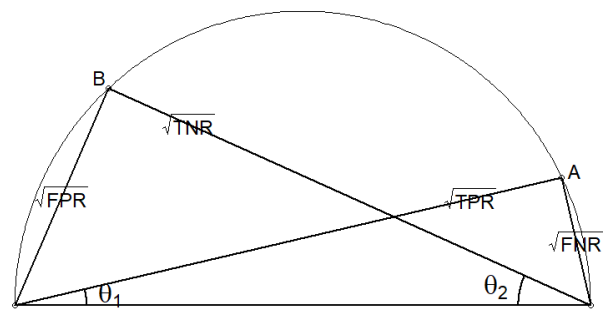

Figure 4: TPR-TNR plot.

\section{Conclusions}

Based on the well-known confusion matrix, two equations whose summation is one are obtained in (2.1). Two right-angled triangles could be imagined using these two equations. These two triangles are drawn on an upper half-circle. The rightmost and leftmost segments of two right-angled triangles are represented as functions of the FNR and FPR, respectively. Since the length of the horizontal line is 1 , then $\theta_{1}$ and $\theta_{2}$ corresponding to the right and left right-angled triangles, respectively, can be defined as $\cos ^{2} \theta_{1}=$ TPR, $\cos ^{2} \theta_{2}=$ TNR. It is found that the TPR, TNR, FPR, and FNR defined in the confusion matrix can be geometrically explained with the TPR-TNR plot proposed in this study.

Points $A$ and $B$ on the TPR-TNR plot denote coordinates in which an upper half-circle meets the right triangle and left triangle, respectively. It is found that point $A$ precedes point $B$. Moreover, the farther point $A$ is away from point $B$, the easier it is to classify the two data sets. Hence, the distance between points $A$ and $B$ plays an important role in determining discriminant power.

Since the TPR and TNR are defined as functions of $\theta_{1}$ and $\theta_{2}$, some optimal thresholds could be found to satisfy three strategies: the first is to maximize the summation of $\cos ^{2} \theta_{1}$ and $\cos ^{2} \theta_{2}$, the second is to maximize the product of $\cos ^{2} \theta_{1}$ and $\cos ^{2} \theta_{2}$, and the last is to minimize the absolute value of the difference between $\cos ^{2} \theta_{1}$ and $\cos ^{2} \theta_{2}$. It is found that these methods discussed three Remarks in Section 3 with similar behavior of accuracy measures such as the Youden index, MVD, SSS, true rate, amended closet-to- $(0,1)$, Kolmogorov-Smirnov statistic, maximizing the AA, and SP. Therefore, we might conclude that various optimal thresholds could be determined using $\theta_{1}$ and $\theta_{2}$ on the TPR-TNR plot.

Various normal distribution functions are considered to derive characteristics of the TPR-TNR plot. We expressed an optimal threshold for each distribution function corresponding TPR-TNR plot geometrically. Hence, the properties of the two angles $\theta_{1}$ and $\theta_{2}$ could be determined using these corresponding TPR-TNR plots.

The TPR-TNR plot describes geometrically various classification rates such as the TPR, FNR, FPR and TNR when a threshold is given. Also this plot can show the variations of these rates as the threshold changes like Figure 2. We might say that this plot can evaluate the classification model similarly to the well known ROC curve using comparison angles and two points in two right angled triangles. Therefore, the TPR-TNR plot proposed in this study can be applied in evaluating classification models when make use the ROC curve together practically and visually. 


\section{References}

Altman DG and Bland JM (1994). Diagnostic tests. 1: Sensitivity and specificity, BMJ: British Medical Journal, 308, 1552.

Bamber D (1975). The area above the ordinal dominance graph and the area below the receiver operating characteristic graph, Journal of Mathematical Psychology, 12, 387-415.

Brasil P (2010). Diagnostic test accuracy evaluation for medical professionals, Package DiagnosisMed in R.

Centor RM (1991). Signal detectability: the use of ROC curves and their analyses, Medical Decision Making, 11, 102-106.

Cho MH and Hong CS (2015). Two optimal threshold criteria for ROC analysis, The Korean Data \& Information Science Society, 26, 255-260.

Connell FA and Koepsell TD (1985). Measures of gain in certainty from a diagnostic test, American Journal of Epidemiology, 121, 744-753.

Egan JP and Egan JP (1975). Signal Detection Theory and ROC-Analysis, Academic Press, New York. Engelmann B, Hayden E, and Tasche D (2003). Testing rating accuracy, Risk, 16, 82-86.

Fawcett T (2004). ROC graphs: Notes and practical considerations for researchers, Machine Learning, 31, 1-38.

Fawcett T (2006). An introduction to ROC analysis, Pattern Recognition Letters, 27, 861-874.

Fluss R, Faraggi D, and Reiser B (2005). Estimation of the Youden Index and its associated cutoff point, Biometrical Journal: Journal of Mathematical Methods in Biosciences, 47, 458-472.

Green DM and Swets JA (1966). Signal Detection Theory and Psychophysics (Vol. 1), Wiley, New York.

Hanley JA and McNeil BJ (1982). The meaning and use of the area under a receiver operating characteristic (ROC) curve, Radiology, 143, 29-36.

Hong CS and Choi SY (2020). Positive and negative predictive values by the TOC curve, Communications for Statistical Applications and Methods, 27, 211-224.

Hong CS, Joo JS, and Choi JS (2010). Optimal thresholds from mixture distributions, The Korean Journal of Applied Statistics, 23, 13-28.

Hong CS and Lee SJ (2018). TROC curve and accuracy measures, The Korean Data \& Information Science Society, 29, 861-872.

Hong CS and Yoo HS (2011). Optimal criterion of classification accuracy measures for normal mixture, Communications for Statistical Applications and Methods, 18, 343-355.

Hsieh F and Turnbull BW (1996). Nonparametric and semiparametric estimation of the receiver operating characteristic curve, The Annals of Statistics, 24, 25-40.

Krzanowski WJ and Hand DJ (2009). ROC Curves for Continuous Data, CRC Press, Boston.

Liu X (2012). Classification accuracy and cut point selection, Statistics in Medicine, 31, 2676-2686.

Metz CE and Kronman HB (1980). Statistical significance tests for binormal ROC curves, Journal of Mathematical Psychology, 22, 218-243.

Moses LE, Shapiro D, and Littenberg B (1993). Combining independent studies of a diagnostic test into a summary ROC curve: data-analytic approaches and some additional considerations, Statistics in Medicine, 12, 1293-1316.

Oehr P and Ecke T (2020). Establishment and characterization of an empirical biomarker SS/PV-ROC plot using results of the UBC@ rapid test in bladder cancer, Entropy, 22, 729.

Pepe MS (2003). The Statistical Evaluation of Medical Tests for Classification and Prediction, Oxford University Press, Oxford. 
Perkins NJ and Schisterman EF (2005). The Youden Index and the optimal cut-point corrected for measurement error, Biometrical Journal: Journal of Mathematical Methods in Biosciences, 47, 428-441.

Perkins NJ and Schisterman EF (2006). The inconsistency of "optimal" cutpoints obtained using two criteria based on the receiver operating characteristic curve, American Journal of Epidemiology, 163, 670-675.

Pontius Jr RG and Si K (2014). The total operating characteristic to measure diagnostic ability for multiple thresholds, International Journal of Geographical Information Science, 28, 570-583.

Provost F and Fawcett T (1997). Analysis and visualization of classifier performance with nonuniform class and cost distributions. In Proceedings of AAAI-97 Workshop on AI Approaches to Fraud Detection \& Risk Management, 57-63.

Provost F and Fawcett T (2001). Robust classification for imprecise environments, Machine Learning, 42, 203-231.

Sonego P, Kocsor A, and Pongor S (2008). ROC analysis: applications to the classification of biological sequences and 3D structures, Briefings in Bioinformatics, 9, 198-209.

Stein RM (2005). The relationship between default prediction and lending profits: Integrating ROC analysis and loan pricing, Journal of Banking \& Finance, 29, 1213-1236.

Swets JA (1988). Measuring the accuracy of diagnostic systems, Science, 240, 1285-1293.

Tasche D (2008). Validation of internal rating systems and PD estimates. In The Analytics of Risk Model Validation (PP. 169-196), Academic Press.

Vuk M and Curk T (2006). ROC curve, lift chart and calibration plot, Metodoloski Zvezki, 3, 89-108. Youden WJ (1950). Index for rating diagnostic tests, Cancer, 3, 32-35.

Zweig MH and Campbell G (1993). Receiver-operating characteristic (ROC) plots: a fundamental evaluation tool in clinical medicine, Clinical Chemistry, 39, 561-577. 
